\title{
Boire et (-)pić (się) : analyse et désambiguïsation
}

\author{
Boire and (-)pić (się): analysis and disambiguation
}

\author{
Joanna Cholewa \\ Université de Białystok \\ j.cholewa@uwb.edu.pl
}

\begin{abstract}
This paper analyses different senses of the French verb boire, especially those that can be paraphrased by 'swallowing liquid', the most easily associated with this verb. To these meanings correspond in Polish the imperfective form pić and some perfective verbs, such as wypić, napić się and przepić. The semantico-syntactic analysis of the verb boire will be followed by the Polish equivalents of each construction. The objective is to observe regularities in the choice of the Polish equivalent of each French structure. The results will be able to enrich the information for bilingual dictionaries and for machine translation.
\end{abstract}

Keywords: analysis, disambiguation, semantico-syntactic construction, equivalent

\section{INTRODUCTION}

Le phénomène de polysémie continue à être non seulement un obstacle pour les travaux destinés à la traduction automatique, mais aussi une des difficultés majeures pour la rédaction des bons dictionnaires bilingues, dont les informations soient suffisantes pour un utilisateur ne connaissant pas bien la langue. Le phénomène en question, propre aux langues naturelles, touche d'une façon particulière le système verbal. Depuis la deuxième moitié du siècle dernier, et ceci tout d'abord sous l'inspiration des besoins de la traduction automatique, les linguistes tentent de proposer un cadre pour la désambiguïsation des verbes. Dans les tables de Maurice Gross (1975), il existe autant de verbes distincts que de constructions syntaxiques différentes, c'est donc un traitement homonymique de la polysémie. Pour Gaston 
Gross $(2012,2015)$, la notion centrale de l'analyse d'une unité polysémique est celle de 'classes d'objets', établies par les besoins de la description des prédicats. Cette analyse base sur les 'emplois', possibles à découvrir au sein d'une phrase. L'approche orientée objets de Wiesław Banyś place aussi au centre de la description linguistique la notion de 'classe d'objets', qui est pour ce linguiste un ensemble d'objets, soit d'unités linguistiques qui partagent un certain nombre d'opérations et d'attributs (Banyś, 2002a et 2002b). Pour Desclés (par exemple 2003, 2005), chaque verbe a une signification de base, appelée invariant ou archétype sémantico-cognitif, qui transcende tous les emplois de celui-ci. Chaque signification d'un verbe polysémique est exprimée par un prédicat avec ses arguments: les constructions syntaxiques encodent la relation prédicative, obtenue par l'application du prédicat à ses arguments. Elle est représentée par un schéma sémantico-cognitif, où l'on observe un agencement de primitives sémantiques dont Desclés établit un répertoire. Dans l'approche continue du sens (Victorri, 2002 ; François, 2006 ; Venant, 2008), se voulant éviter les difficultés qu'engendrent les représentations discrètes, à toute unité polysémique est associé un espace sémantique (représentation graphique des synonymes d'un mot, qui permet rapidement de se rendre compte de ses différents sens). Tenant compte de ces approches, plusieurs travaux ont vu le jour, proposant la désambiguïsation de certains verbes, par exemple Cholewa (2008, 2017), Emirkanian (2008), Hrabia (2014), Jalenques (2010), Leemann (2004), Vandeloise (2007), Victorri (2010), Yune (2011), Żłobińska-Nowak (2008).

Nous sommes d'accord avec la constatation que c'est dans la phrase que l'on découvre de quel emploi d'un mot polysémique il s'agit, l'emploi étant un prédicat défini par ses arguments, et qui, à son tour, délimite le champ des arguments (Gross, 2015). Les arguments seront décrits à l'aide des classes sémantiques (animé, humain, concret, abstrait), et des sous-catégories, levant les ambiguités à l'intérieur de ces hyper-classes. Ces sous-catégories sont établies (comme les classes d'objets de Gross, 1999) compte tenu des besoins de la description des prédicats choisis. L'analyse tiendra également compte du contexte dans lequel apparaît un emploi donné.

Nous voulons, dans ce qui suit, procéder à une désambiguïsation du verbe boire, et proposer à ses différents emplois les équivalents polonais. Nous limitons volontairement notre analyse à ceux des sens de boire que l'on peut résumer par 'avaler, consommer du liquide, en parlant d'un être animé'. Ainsi, en seront exclues les acceptions suivantes de boire, distinguées dans le $\mathrm{TLFi}^{1}$ :

- par analogie, avec le sujet désignant un corps perméable ou poreux, 'absorber un liquide ; se laisser pénétrer, imprégner par lui' ;

- au sens figuré, avec le sujet désignant généralement une personne :

\footnotetext{
${ }^{1}$ Trésor de la Langue Française informatisé, http://atilf.atilf.fr/.
} 
a) 'recevoir un bien d'ordre physique, moral ou intellectuel et en jouir ou en tirer parti intensément' : C'est à la vraie source de sa vie que son âme va boire ;

b) 'surmonter une difficulté' : Cheval qui boit l'obstacle 'qui le franchit très facilement';

c) 'supporter quelque chose de pénible, d'humiliant' : Boire l'amertume, un affront, la honte.

Notre objectif est de constater des régularités dans le choix du verbe polonais lors de la recherche d'un équivalent.

\section{DÉFINITIONS DE BOIRE ET DE SES ÉQUIVALENTS POLONAIS LES PLUS FRÉQUENTS}

\subsection{Boire}

Le verbe transitif boire, utilisé avec le sujet désignant une personne ou un animal et signifiant 'avaler un liquide', entre (selon TLFi) dans deux types de constructions :

1) soit le complément d'objet direct est exprimé : boire de l'eau, un verre;

2) soit boire apparaît dans l'emploi absolu :

- le complément non exprimé peut désigner toute espèce de boissons : boire chaud, frais, à longs traits; boire à sa soif ; boire dans un verre; verser à boire ;

- le complément non exprimé se réfère à toutes sortes de boissons alcoolisées; boire signifie alors 'avoir coutume d'en boire avec excès, être alcoolique'.

Le dictionnaire Les verbes français $\left(\mathrm{LVF}^{2}\right)$ délimite et divise différemment les sens de boire. Il en distingue quatre, dont un est un emploi passif :

- boire 1 - 'chopiner, zinguer' : On boit de l'eau, Ce vin se boit facilement, On boit beaucoup l'été,

- boire 2 - 'se soûler': On boit depuis l'adolescence, On boit de l'alcool,

- boire 4 (être bu) - 'être ivre' : On est bu après cette réunion.

Le sens numéro 3, avec le sujet non animé, se trouvera hors de notre analyse :

- boire 3 - 's'imbiber, s'imprégner': Cette éponge boit bien, Le buvard boit l'encre.

Nous pouvons constater que LVF fait la différence entre 'boire un peu, n'importe quelle boisson' (boire 1) et 'boire excessivement, en parlant de l'alcool,

\footnotetext{
${ }^{2}$ Les verbes français de Jean Dubois et Françoise Dubois-Charlier (Version LVF +1 ), http://rali. iro.umontreal.ca/rali/?q=fr/lvf.
} 
se soûler' (boire 2), emplois qui sont confondus dans TLFi. Par contre, LVF met ensemble les emplois avec le complément d'objet direct exprimé et les emplois absolus, que le TLFi sépare.

\subsection{Les équivalents polonais de boire}

La plupart des infinitifs polonais apparaissent sous au moins deux formes morphologiquement distinctes, appelées dans la tradition grammaticale 'perfective' et 'imperfective'. Quand on cherche les correspondants polonais des sens mentionnés de boire dans le dictionnaire bilingue $\left(\mathrm{WSFP}^{3}\right)$, on s'aperçoit de la présence d'un couple imperfectif / perfectif de base, pić / wypić. Dans l'un des exemples, à côté de wypić, apparaît napić się : boire tout son soûl - 'wypić, ile można, napić się' (ainsi que d'autres verbes, mais qui traduisent des locutions figées, comme par exemple boire à sa soif - 'zaspokoić pragnienie'). Pić, wypić et napić się semblent essentiels pour traduire boire. Pourtant, il convient de noter que le dictionnaire Słownik gniazd słowotwórczych współczesnego języka ogólnopolskiego (Skarżyński, 2004, p. 43-45) distingue plusieurs autres verbes, construits sur la base pić par l'ajout d'un préfixe : opić, przepić, rozpić, spić (się), upić (się), zapić, ainsi que des imperfectifs, formés sur les dérivés préfixaux perfectifs par remplacement de formant, comme par exemple przepijać, wypijać (Wróbel (2001) parle dans ce cas de la dérivation aspectuelle du deuxième type). La préfixation constitue une des caractéristiques de base de la formation des verbes en polonais. Ajoutés à la base verbale, les préfixes modifient son contenu sémantique ou y apportent des éléments nouveaux, mettant l'accent par exemple sur le début, la fin, la répétitivité, l'intensité, le résultat de l'action (Wróbel, 2001 ; Włodarczyk et Włodarczyk, 2001). C’est donc un procédé complexe, résultant en une multiplicité de combinaisons sémantiques, qui servent à exprimer les valeurs aspectuelles que la langue française transmet principalement grâce à l'emploi des modes et des temps.

Nous sommes persuadée qu'il est possible de proposer des schémas sémanticosyntaxiques qui, malgré cette complexité des systèmes verbaux des deux langues, faciliteront le choix des correspondants polonais de boire, et seront peut-être applicables pour la traduction de ce verbe français dans d'autres langues.

Pour pouvoir comparer les sens sélectionnés de boire à ceux de ses équivalents polonais, nous utilisons le dictionnaire Wielki słownik języka polskiego (WSJP ${ }^{4}$ ). Seront ici présentées six formes polonaises : pić, wypić, wypijać, napić się, przepić et przepijać. Chacun de leur sens est illustré par des collocations, ainsi que par un schéma syntaxique.

\footnotetext{
${ }^{3}$ Wielki stownik francusko-polski.

${ }^{4}$ Wielki słownik języka polskiego, http://www.wsjp.pl/.
} 
1. Pić, dans le sens 'avaler un liquide', est représenté par deux sens :

a) 'nabierać płyn do ust i połykać go' ('prendre du liquide dans sa bouche et l'avaler') :

- pić herbatę, kawę; wódkę, piwo ('boire du thé, du café ; de la vodka, de la bière') ;

- pić małymi tykami ('boire à petits traits') ;

- pić przez słomkę ('boire avec une paille') ;

- pić coś $w$ bufecie, $w$ domu ('boire qqc. au buffet, à la maison') ;

- pić z butelki, z filiżanki, z kubka ('boire dans une bouteille, dans une tasse, dans un bol') ;

- pić chciwie, dużo, powoli ('boire avidement, beaucoup, lentement') ;

- schéma syntaxique : N[animé] + pić + N1;

b) 'spożywać alkohol, zwłaszcza w nadmiarze' ('consommer de l'alcool, surtout excessivement') :

- mą̇, ojciec pije; pić $w$ barze, $w$ pracy; pić z kolegami ('le mari, le père boit ; boire dans un bar, au travail ; boire avec des copains') ;

- schéma syntaxique : N[animé] + pić.

2. Napić się est un verbe perfectif, formé sur pić par l'adjonction de na-, préfixe dont le sens implique fortement la résultativité (Giermak-Zielińska, 1979). Selon Wróbel (2001), il peut aussi modifier le contenu sémantique de la base par une caractéristique quantitative, se référant à l'addition des portions de l'action principale (par exemple, znosić krzesła - 'apporter des chaiser', na-znosić krzeset 'apporter / procurer beaucoup de chaises'). D'après le WSJP, napić się signifie :

a) 'nabrać do ust i połknąć pewną ilość płynu' ('prendre dans sa bouche et avaler une certaine quantité de liquide') :

- napić się herbaty, kawy; wódki, piwa, tyka czegoś ('boire du thé, du café ; de la vodka, de la bière, une gorgée de qqc.') ;

- napić się z dzbana, ze szklanki ('boire dans une cruche, dans un verre') ;

- napić się trochę, do woli ('boire un peu, à satiété') ;

- chcieć, móc, musieć, postanowić napić się ('vouloir, pouvoir, devoir boire, décider de boire') ;

- napić się i pogadać, napić się i zjeść; wstać i napić się ('boire et parler, boire et manger ; se lever et boire') ;

- schéma syntaxique : N1[animé] + napić się + N1;

b) 'spożyć alkohol' ('consommer de l'alcool') :

- napić się $w$ barze, $w$ pracy, $w$ czyimś towarzystwie ('boire dans un bar, au travail ; en compagnie de qqn') ;

- napić się na weselu, na zgodę ('boire à la noce ; pour se réconcilier') ;

- napić się za spotkanie, za czyjeś zdrowie ('boire à la rencontre, à la santé de qqn') ;

- napić się z kimś ('boire avec qqn') ; 
- napić się i tańcować, napić się $i$ zasnać ('boire et danser; boire et s'endormir') ;

- schéma syntaxique : N0[humain] + napić się.

3. La deuxième forme perfective, wypić, est construite par l'adjonction du préfixe $w y$ - à la base pić. $W y$-implique une action réalisée dans sa totalité (GiermakZielińska, 1979 ; Janowska, 1999). Dans le même esprit, Wróbel (2001) affirme que wy- apporte à la base une modification, en précisant que l'action exprimée par le verbe concerne tous les objets appartenant à un groupe (par exemple : łapać muchy'attraper des mouches', wy-łapać muchy - 'attraper toutes les mouches'). Il peut aussi conférer une nuance d'intensité dans le cas des formations affectives-résultatives (par exemple wytańczyć się - 'danser à sa guise, à volonté'), mais ce n'est pas le cas du verbe que nous analysons. Dans le dictionnaire WSJP, wypić signifie :

a) 'spożyć do końca porcję napoju' ('consommer toute la portion d'un liquide') : - wypić alkohol, koniak, piwo, herbate, kawę, mleko, wodę ('boire l'alcool, le cognac, la bière, le thé, le café, le lait, l'eau') ;

- wypić butelkę, filiżanke, kieliszek, łyk, zawartość czegoś ('boire une bouteille, une tasse, un verre, une gorgée, le contenu de qqc.') ;

- wypić chciwie, łapczywie, trochę; duszkiem, jednym haustem, matymi bykami, $w$ milczeniu ('boire goulûment, avidement, un peu ; d'un trait, d'un seul coup, à petites gorgées, dans le silence') ;

- można wypić ('on peut boire') ;

- wypić coś i pogadać; zjeść i wypić ('boire quelque chose et parler ; manger et boire') ;

- schéma syntaxique : N0[animé] + wypić + N1;

b) 'spożyć alkohol' ('consommer de l'alcool') :

- wypić dla odwagi ('boire pour le courage') ;

- wypić na bankiecie ('boire pendant une réception') ;

- wypić za przyjaźń, za czyjeś zdrowie ('boire à l'amitié, à la santé de qqn') ;

- wypić dużo, porzadnie, troszke ('boire beaucoup, considérablement, un peu') ;

- lubić, musieć, zamierzać wypić ('aimer, devoir, aller boire') ;

- wypić $i$ wypalić, wypić i zakasić, wypić i zabawić się ('boire et fumer, boire et manger, boire et s'amuser') ;

- schéma syntaxique : N0[animé] + wypić.

Le deuxième sens de chacun des verbes évoqués est le même : 'consommer de l'alcool'; les deux sont liés à la deuxième acception de l'imperfectif pić, même si les exemples qui les illustrent ne se ressemblent plus. Par contre, le premier sens de chacun des perfectifs, résultant du préfixe utilisé, est différent : wypić est focalisé sur 'boire jusqu'à la fin toute une portion d'un liquide' (l'action décrite par la base est réalisée dans sa totalité) et napić się sur 'avaler une certaine quantité d'un liquide', cette quantité étant souvent précisée par le nom en position de N1. 
4. Wypijać, formé sur wypić, témoigne de la dérivation aspectuelle du deuxième type (Wróbel, 2001) : il s'agit de la construction d'un correspondant imperfectif du verbe perfectif préfixé. Ce type de dérivation consiste à substituer le formant caractéristique pour les verbes imperfectifs (-a-/-aj- est le plus fréquent) à celui de la forme perfective. Ainsi, pour wypijać, $-a-/-a j$ - se substitue à $-i-/-\varnothing-$, présent dans wypić : wypi-ø-ć (wypi-j-a: forme conjuguée) $\rightarrow$ wypij-a-ć (wypij-aj-a : forme conjuguée) (Grzegorczykowa, Laskowski, Wróbel, 1998; Wróbel, 2001). Wypijać signifie 'spożywać napój porcjami lub do końca', 'consommer une boisson par portions ou jusqu'à la fin' :

- wypijać alkohol, herbatę, kawę, koniak ('boire l'alcool, le thé, le café, le cognac'); wypijać butelkę, filiżankę, kieliszek, kubek, tyk ('boire une bouteille, une tasse, un verre, un bol, une gorgée') ;

- wypijać coś na przyjęciu, na śniadanie ('boire qqc. à une réception, à un déjeuner') ;

- wypijać codziennie, szybko, trochę; jednym haustem, na czczo, od czasu do czasu, z pośpiechem ('boire chaque jour, vite, un peu ; d'un trait, à jeun, de temps en temps, à la hâte').

5. Przepić (perfectif) est construit sur pić par l'adjonction du préfixe prze-, qui signifie une perte d'un objet à la suite de l'action de base (Wróbel, 2001). Dans przepijać (imperfectif), le formant -a-/-aj- se substitue à $-i-/-\varnothing-$, comme dans le cas de wypić / wypijać. Ainsi, de przepi-ø-ć (przepi-j-a), est dérivé przepij-a-ć (przepijaj-a). Przepić / przepijać possèdent quatre sens, liés à l'action de boire, même si ce lien s'avère indirect, comme dans les sens 1, 2 et 3 ci-dessous :

a) pot. 'wydać wszystkie pieniądze lub oddać coś wartościowego, aby zdobyć alkohol i upić się nim' ('dépenser tout l'argent ou donner quelque chose de précieux pour obtenir de l'alcool et s'enivrer') : przepić pieniadze, odprawe, rentę, majątek ('croquer/ dépenser l'argent, l'indemnité de départ, la rente, les biens dans la boisson') ;

b) 'spędzić pewien określony czas na piciu alkoholu' ('passer un temps à boire de l'alcool') : przepić (cały) dzień, (cała) noc, 11 lat, pót życia ('passer (toute) une journée, (toute) une nuit, 11 ans, la moitié de sa vie à boire') ;

c) 'podczas spotkania towarzyskiego zwrócić się bezpośrednio do jednej z osób, wypowiadając najczęściej formułę wyrażającą życzenie, aby ta osoba była zdrowa, i wypić zaraz potem porcję alkoholu' ('pendant un repas convivial, s'adresser directement à l'une des personnes, en prononçant le plus souvent une formule qui exprime les vœux de bonne santé, et boire juste après un verre d'alcool') : przepić do kogoś ('trinquer avec qqn');

d) 'wypić coś zaraz po przełknięciu czegoś innego lub zjedzeniu czegoś' ('boire qqc. juste après avoir bu ou mangé qqc.') : przepić coś herbata, oranżada, wódka ('boire du thé, de la limonade, de la vodka après avoir bu ou mangé qqc.'), 
przepić czymś positek, danie ('boire qqc. après un repas, après avoir mangé un plat').

Il est clair, quand on regarde les verbes polonais sélectionnés pour l'analyse, que leurs sens résultent de celui de la base, mais aussi de ceux des préfixes, qui n'ont pas que la fonction aspectuelle grammaticale de perfectivation. Ainsi, pić désigne l'action principale de boire du liquide, alors que les préfixes apportent des précisions, en modifiant le contenu sémantique de la base pić : le préfixe $n a$ - met l'accent sur la quantité et/ou le résultat, wy- sur l'action réalisée dans sa totalité et prze-sur la perte d'un objet à la suite de l'action de base.

\section{CONSTRUCTIONS SÉMANTICO-SYNTAXIQUES DE BOIRE ET LEURS ÉQUIVALENTS POLONAIS}

Dans cette section, nous allons distinguer différents emplois de boire, tenant compte de la construction syntaxique du verbe, des propriétés sémantiques de ses arguments, ainsi que du contexte. Dans les exemples qui les illustrent, tirés de Frantext ${ }^{5}$, boire apparaît dans différentes formes. Nous les avons sélectionnées de façon à avoir, pour chaque emploi, des exemples traduits ${ }^{6}$ en polonais par une forme imperfective, et ceux auxquels correspond un verbe perfectif. Le but en était de démontrer que pour les schémas sémantico-syntaxiques de boire, il est possible de proposer des couples imperfectif/ perfectif polonais équivalents. Notre objectif n'est pas de donner toutes les variantes de traduction des exemples sélectionnés, mais d'indiquer celle qui est la plus vraisemblable.

\section{1. $\mathrm{N}_{[\text {HUMAIN }]}+$ boire $\rightarrow$ pić / wypić / napić się}

Pour boire sans complément exprimé, le polonais présente le choix entre l'imperfectif pić et les formes perfectives wypić et napić się. Ainsi, on traduira par pić les exemples où boire exprime une valeur inachevée :

- Ils font semblant de boire, de reposer leurs verres et de payer ('Udają, że piją, odstawiają kieliszki i płacą');

- On boit, ou on cause - demande Armoire ('Pijemy czy rozmawiamy - pyta Armoire') ;

- Jouons, buvons et tirons-nous ('Bawmy się, pijmy i zwiewajmy').

Mais certains emplois à valeur achevée peuvent être aussi traduits par pić, à condition qu'il s'agit de boire de l'alcool :

\footnotetext{
${ }^{5} \mathrm{https}$ ://www.frantext.fr/.

${ }^{6}$ Les exemples français sont traduits par l'auteur.
} 
- Aujourd'hui, il a bu. Chaque vendredi, il boit ('Dzisiaj pił. Pije w każdy piątek') ;

- «Tu as bu? ? «Oui, j'ai bu » ('Piłeś ?' - 'Tak, piłem').

Les formes perfectives wypić et napić się peuvent commuter quand le contexte indique qu'il s'agit de l'alcool :

- Ont assez bu comme ça. Sont à la bourre ('Dość już wypili / się napili. Są nachlani');

- Quand il a bu, elle le voit de loin ('Kiedy wypije / się napije, ona od razu to widzi / wie').

Si le contexte refuse qu'il puisse s'agir de l'alcool, l'usage de wypić est injustifié : en effet, si la boisson n'est pas alcoolisée, wypić signifierait 'consommer toute la portion d'un liquide'. Pour l'exemple qui suit, le polonais devrait alors sélectionner napić się:

- Danse devant la fontaine. Penche-toi pour boire ('Tańcz przed fontanną. Schyl się, żeby się napić').

Le schéma sémantico-syntaxique que nous envisageons ne suffit pas pour désambiguïser le verbe français. Il est nécessaire de connaître le contexte afin de sélectionner le perfectif polonais. Deux schémas devraient donc être formulés :

$-\mathrm{N} 0_{\text {[humain] }}+$ boire [liquide, alcool] $\rightarrow$ pić / wypić / napić się ;

$-\mathrm{N}_{\text {[humain] }}+$ boire [liquide, non alcool] $\rightarrow$ pić / napić się.

La difficulté pour la traduction consiste en ce que c'est le contexte qui impose le choix d'un équivalent polonais perfectif, en indiquant si le complément non exprimé se réfère à une boisson alcoolisée ou non : boire peut être traduit par wypić / napić się (et parfois aussi par l'imperfectif pić) quand il s'agit de l'alcool, et par napić się quand il s'agit d'une boisson non alcoolisée.

\section{2. $\mathrm{N}_{[\text {HUMAIN }]}+$ boire $+\mathrm{N} 1_{[\mathrm{LIQUIDE}]} \rightarrow$ pić / wypić / napić się}

Boire avec le complément d'objet direct [liquide] ouvre la possibilité de sélectionner en polonais pić / wypić et napić się, comme dans la section précédente. Ainsi, l'imperfectif pić est utilisé pour exprimer une action inachevée :

- Je buvais une bière avec Walter qui me faisait la cour ('Piłam piwo z Walterem, który się do mnie zalecał') ;

- Je bois du lait avec une goutte de café ('Piję mleko z odrobiną kawy');

- Elle boit le cidre à petites gorgées ('Piję małymi łykami cydr').

Le choix entre les perfectifs wypić et napić się est plus évident que pour l'emploi absolu de boire. La langue polonaise sélectionne le verbe compte tenu du déterminant qui précède N1. Ainsi, la construction française boire + article partitif + N1 [liquide] sera traduite en polonais par napić się : 
- On va boire du chocolat très épais qui mousse ('Napijemy się gęstej, musującej czekolady');

- Les enfants burent de la limonade ('Dzieci napiły się lemoniady');

- Enfin je bus du cognac, cela me calma un peu ('W końcu napiłem się koniaku, co trochę mnie uspokoiło');

- Betty avait bu du vin ('Betty napiła się wina').

La définitude bloque napić się, et autorise wypić :

- Madame Jeunehomme but son café en deux gorgées ('Pani Jeunehomme dwoma łykami wypiła swoją kawę');

- J'ai bu mon eau ('Wypiłem wodę');

- Je bus le café debout, les jambes légèrement écartées ('Wypiłem kawę na stojąco, z lekko rozstawionymi nogami') ;

- Je bus avidement le jus d'orange que m'apporta Hélène ('Chciwie wypiłem sok pomarańczowy, który przyniosła mi Hélène').

Enfin, boire suivi de l'article indéfini ouvre le choix entre wypić et napić się :

- Inconcevable à leurs yeux [...] de boire une boisson froide ('W ich oczach jest niepojęte, aby napić się czegoś / wypić coś zimnego');

- Tu bois un café avec nous ? ('Napijesz się z nami kawy ? / Wypijesz z nami kawę ?');

- Il avait envie de s'asseoir dans un café, de boire une bière et de fumer une cigarette ('Miał ochotę usiąść w kawiarni, wypić piwo / napić się piwa i zapalić papierosa').

Dans ces exemples, un / une peuvent être considérés soit comme articles indéfinis, soit comme adjectifs numéraux exprimant une quantité. Dans le groupe suivant, où le N1[liquide] est suivi de l'adjectif épithète, un / une expriment une quantité : un café réparateur signifie une [tasse de] café réparateur, un punch délicieux - un [verre de] punch délicieux, et un rhum blanc - un [verre de] rhum blanc. Dans la traduction apparaît alors obligatoirement une expression de quantité appropriée au type de boisson, et boire est traduit par wypić :

- Elle fonça au Montana pour boire un café réparateur ('Pobiegła do hotelu [Montana], żeby wypić filiżankę krzepiącej kawy');

- Olga, désespérée, qui a bu un rhum blanc avec nous ('Zrozpaczona Olga, która wypiła z nami kieliszek białego rumu');

- On dansera et on boira un punch délicieux, confectionné par nos soins ('Zatańczymy i wypijemy po kieliszku pysznego ponczu, starannie przez nas sporządzonego').

Dans ce dernier exemple, 'napijemy się po kieliszku' semble aussi autorisé, à notre avis, compte tenu du sujet qui renvoie à une pluralité.

Concernant cette construction, nous pouvons résumer que boire exprimant l'inachèvement se fait correspondre en polonais la forme imperfective pić. Dans le cas de l'action achevée, le choix entre wypić et napić się est corrélé avec le déterminant en français. Ainsi, si N1 est précédé de l'article partitif, boire fait 
sélectionner napić się. S'il est précédé d'un déterminant défini, c'est le verbe wypić qui s'utilise en polonais. Enfin, l'article indéfini placé devant N1 laisse le choix entre wypić et napić się.

\section{3. $\mathrm{N}_{\text {[HUMAIN] }}+$ boire $+\mathrm{N}_{\text {[QUANT.] }} \mathrm{deN} 1_{\text {[LIQUIDE] }} \rightarrow$ pić / wypijać / wypić / napić się}

Comme l'indique le dictionnaire, si N1 est précédé d'une expression de quantité, c'est le verbe wypić qui s'emploie (sens 1: 'consommer toute la portion d'un liquide' : wypić butelkę, filiżankę, kieliszek, tyk, zawartość czegoś, 'boire une bouteille, une tasse, un verre, une gorgée, le contenu de qqc'). Ainsi, nous allons traduire boire par wypić dans les exemples suivants :

- Puis je bus un verre d'eau froide ('Potem wypiłem szklankę zimnej wody');

- Ils burent encore un verre de prunelle ('Wypili jeszcze kieliszek śliwowicy') ;

- Je bus une lampée de brandy ('Wypiłem łyk brandy') ;

- Je ne boirai plus une goutte de calvados de ma vie ('Już nigdy w życiu nie wypiję kropli calvadosu');

- Babé pencha la tête en arrière pour boire la dernière goutte de café ('Babé przechylił głowę w tył, żeby wypić ostatnią kroplę kawy') ;

- Lucille prit la tasse entre ses deux mains, but une gorgée de café ('Lucille wzięła filiżankę w obie ręce, wypiła łyk kawy') ;

- Mes deux grands-pères avaient ensemble bu beaucoup de vin ('Obaj moi dziadkowie wypili razem dużo wina')

Quand N1 est précédé de un peu, deux verbes sont possibles à l'aspect perfectif wypić et napić się:

- Alors je bus un peu de cognac ('Napiłem się / wypiłem więc trochę koniaku');

- Ils burent un peu de vodka, et même beaucoup ('Napili się / wypili trochę wódki, nawet sporo');

- Lucille s'interrompit, but un peu de café et s'essuya les yeux ('Lucille przerwała, wypiła / napiła się trochę kawy i przetarła oczy').

À l'aspect imperfectif, la construction boire $+\mathrm{N}_{\text {[quant.] }} \mathrm{deN} 1_{\text {[liquide] }}$ se traduit en polonais par le verbe pić :

- Maman et nous buvions notre tasse de thé ('Piliśmy z mamą po filiżance herbaty') ;

- Sa chambre, où les volets étaient fermés et où elle buvait une tasse de thé dans son lit ('Jej pokój, gdzie żaluzje były zamknięte i gdzie piła w łóżku filiżankę herbaty').

Le quantitatif un peu permet de substituer wypijać à pić :

- Je bois juste un peu de vin rouge ('Piję / wypijam troszeczkę czerwonego wina');

- Des dames grignotent des gâteaux et boivent un peu de thé ('Panie skubią ciastka i piją / wypijają troszkę herbaty'). 
Même si wypijać est expliqué par 'spożywać napój porcjami lub do końca' ('consommer une boisson par portions ou jusqu'à la fin'), ce verbe a le plus souvent une valeur aspectuelle d'itérativité. Ainsi, nous traduirons :

- Il en [du café] buvait tous les matins une ou deux tasses ('Każdego ranka pił / wypijał jedną lub dwie filiżanki [kawy]');

- Je bois plus de bière à l'étranger ('Piję / wypijam więcej piwa za granicą').

\section{4. $\mathrm{N}_{[\text {HUMAIN }]}+$ boire $+\mathrm{N}_{[\text {[QUANT.] }} \rightarrow$ pić / wypijać / wypić}

$\mathrm{N} 1_{\text {[quant.] }}$ utilisé par métonymie (le contenant pour le contenu) après boire mène à un blocage de napić się au perfectif, laissant comme seule solution wypić :

- Armoire, qui dans l'intervalle a bu une gorgée, me tend la bouteille ('Armoire, który w międzyczasie wypił łyk, wyciąga do mnie butelkę') ;

- Ils burent quelques gorgées lentement, en se regardant par-dessus le verre ('Wypili wolno kilka łyków, patrząc na siebie ponad kieliszkami');

- Il me reste quelques bonnes bouteilles. Nous devons les boire, puisque, de toute façon, la cave sera cambriolée ('Zostało mi kilka dobrych butelek. Musimy je wypić, bo i tak piwnica zostanie obrabowana') ;

- Je bus ainsi plusieurs verres, cela me ramenait à la vie (' $\mathrm{W}$ ten sposób wypiłem kilka kieliszków, to przywracało mnie do życia').

À l'imperfectif, à côté de pić apparaît wypijać. Il se substitue à pić quand le contexte indique qu'il s'agit soit de l'itérativité ('consommer une boisson par portions') :

- À neuf ans il buvait son litre par jour et bien souvent son litre et demi ('W wieku dziewięciu lat wypijał / pił litr dziennie, a często półtora litra') ; soit de la valeur terminative ('consommer une boisson jusqu'à la fin') :

- Il boit une gorgée, goulument ('Wypija / pije łapczywie łyk') ;

- Mais non, c'est de la folie, personne ne boit un demi-litre comme ça ('No nie, to szaleństwo, nikt nie wypija / pije ot tak pół litra');

- Eh, bois pas tout... laisse-m'en une goutte! ('Ej, nie wypijaj / pij wszystkiego... zostaw mi kropelkę!').

Mais wypijać n'est plus possible quand la valeur itérative ou terminative disparaît :

- J'buvais une chope, $t$ 'sais avec qui ? Ma-ry-se! ('Piłem / *wypijałem kufelek, wiesz z kim? Z Ma-ry-se!').

\section{5. $\mathrm{N}_{[\text {HUMAIN] }}+$ boire à $+\mathrm{N}_{[\text {ABSTRAIT / HUMAIN }]} \rightarrow$ pić / wypić za / napić się za}

La construction avec le complément prépositionnel boire à se traduit en polonais par pić à l'imperfectif et wypić / napić się (sans restrictions) au perfectif : 
- Je bois à ceux que nous aimons ('Piję za tych, których kochamy');

- Il avait pris l'habitude de boire à la paix ('Nabrał zwyczaju, żeby pić za pokój') ;

- Et buvons à ton succès ! ('(Wy)pijmy / napijmy się za twój sukces’);

- Ils burent donc à la santé de Rodney ('Wypili / napili się więc za zdrowie Rodneya').

\section{6. $\mathrm{N}_{[\text {HUMAIN }]}+$ boire $+\mathrm{N}_{[\text {[ABSTRAIT }]} \rightarrow$ przepijać / przepić}

Le dernier emploi que nous allons analyser est boire construit avec N1[abstrait]. Il n'est pas très fréquent en français (en effet, nous en avons trouvé une occurrence parmi environ quinze mille pour boire). Dans le schéma présenté, le nom figurant en position de N1 témoigne d'une métonymie, qui consiste en ce que la boisson est remplacée par l'argent dépensé pour l'acheter :

- Il avait bu l'argent des terres vendues ('Przepił pieniądze ze sprzedanej ziemi').

Nous avons en plus trouvé un exemple sur Internet :

- boire sa paye ('przepić wypłatę').

Utilisé avec N1[abstrait] appartenant à la classe 'argent', boire est traduit en polonais par un couple przepijać / przepić, dont la fréquence est beaucoup plus élevée. Le dictionnaire WSJP propose, comme nous l'avons dit dans la section 2.2.5 (sens 1), quatre collocations : przepić pieniadze, odprawę, rentę, majątek ('croquer / dépenser l'argent, l'indemnité de départ, la rente, les biens dans la boisson'). Dans le corpus NKJP (Narodowy Korpus Języka Polskiego ${ }^{7}$ ), nous pouvons en trouver d'autres, dont une partie se réfère à l'argent (przepić pożyczkę, każdy grosz, zarobek, cała sumę, zaliczkę, tapówki - en traduction littérale 'boire le prêt, chaque sou, le gain, toute la somme, les arrhes, les dessous-de-table'), mais aussi aux entités concrètes : przepić firmę, konie, wóz, uprzą̇, portki, telefon ('croquer / dépenser la firme, les chevaux, la charrette, le harnais, son froc, le téléphone dans la boisson'). Dans ce cas, la métonymie caractéristique pour cet emploi est double : boisson $\rightarrow$ argent dépensé pour l'acheter $\rightarrow$ objet vendu pour avoir de l'argent.

\section{CONCLUSION}

La notion d'emploi s'avère efficace pour procéder à une désambiguïsation préliminaire du verbe boire, mais les schémas sémantico-syntaxiques que nous avons distingués en analysant le verbe français ne sont pas systématiquement

\footnotetext{
${ }^{7}$ http://nkjp.pl/
} 
suffisants pour sélectionner l'équivalent polonais. D'autre côté, les traductions de boire par pić, wypić et napić się, suggérées par le grand dictionnaire bilingue (WSFP) ne sont pas satisfaisantes non plus.

Les six verbes polonais que nous avons indiqués dans la section 2.2 se répartissent de façon à ce qu'à chacun des emplois de boire correspondent aussi bien des formes imperfectives que celles perfectives, leur choix étant lié à plusieurs facteurs. Le premier est bien sûr le schéma sémantico-syntaxique de base et les traits sémantiques des noms en position de N1. Pourtant, ce critère est loin d'être suffisant, d'autres facteurs étant la valeur aspectuelle du verbe français, le déterminant qui précède $\mathrm{N} 1$, l'adjectif qui suit $\mathrm{N} 1$, le contexte, et l'apparition de un peu devant $\mathrm{N} 1$.

Ainsi, il faudrait proposer des schémas plus détaillés pour désambiguïser boire :

- $\mathrm{N}_{\text {[humain] }}+$ boire (cf. 3.1)

pour la valeur inachevée de boire :

$\mathrm{NO}_{\text {[humain] }}+$ boire $\rightarrow$ pić

pour la valeur achevée de boire :

$\mathrm{N} 0_{\text {[humain] }}+$ boire (contexte : liquide, alcool) $\rightarrow$ wypić / napić się

$\mathrm{N} 0_{[\text {humain] }}+$ boire $($ contexte $:$ liquide, non alcool) $\rightarrow$ napić się

- $\mathrm{N}_{\text {[humain] }}+$ boire $+\mathrm{N} 1_{\text {[liquide] }}$ (cf. 3.2)

pour la valeur inachevée de boire :

$\mathrm{N} 0_{[\text {humain] }}+$ boire $+\mathrm{N}_{\text {[liquide] }} \rightarrow$ pić

pour la valeur achevée de boire :

$\mathrm{N} 0_{\text {[humain] }}+$ boire + art. partitif $+\mathrm{N}_{\text {[liquide] }} \rightarrow$ napić sie

$\mathrm{N} 0_{\text {[humain] }}+$ boire + art. défini / adj. possessif $+\mathrm{N}_{\text {[liquide] }} \rightarrow$ wypić

$\mathrm{N} 0_{[\text {humain] }}+$ boire + art. indéfini $+\mathrm{N1}_{[\text {liquide] }} \rightarrow$ wypić / napić się

$\mathrm{N}_{[\text {[humain] }}+$ boire + art. indéfini $+\mathrm{N1}_{\text {[liquide] }}+$ adj. épithète $\rightarrow$ wypić

- $\mathrm{N}_{\text {[humain] }}+$ boire $+\mathrm{N}_{\text {[quant.] }} \mathrm{deN} 1_{\text {[liquide] }}$ (cf. 3.3)

pour la valeur inachevée de boire :

$\mathrm{N}_{\text {[humain] }}+$ boire $+\mathrm{N}_{\text {[quant.] }} \mathrm{deN} 1_{\text {[liquide] }} \rightarrow$ pić

$\mathrm{N}_{\text {[humain] }}+$ boire + un peu deN1 $1_{\text {[liquide] }} \rightarrow$ pić / wypijać

pour la valeur itérative de boire :

$\mathrm{N}_{\text {[humain] }}+$ boire + un peu deN1 $1_{\text {[liquide] }} \rightarrow$ pić / wypijać

pour la valeur achevée de boire :

$\mathrm{NO}_{\text {[humain] }}+$ boire $+\mathrm{N}_{\text {[quant.] }} \mathrm{deN} 1_{\text {[liquide] }} \rightarrow$ wypić

$\mathrm{N} 0_{\text {[humain] }}+$ boire + un peu deN1 $1_{\text {[liquide] }} \rightarrow$ wypić / napić sie

- $\mathrm{N}_{\text {[humain] }}+$ boire $+\mathrm{N}_{\text {[quant.] }}$ (cf. 3.4)

pour la valeur inachevée de boire :

$\mathrm{N} 0_{\text {[humain] }}+$ boire $+\mathrm{N1}_{\text {[quant.] }} \rightarrow$ pić

pour la valeur itérative ou terminative de boire :

$\mathrm{N} 0_{\text {[humain] }}+$ boire $+\mathrm{N}_{\text {[quant.] }} \rightarrow$ pić / wypijać

pour la valeur achevée de boire :

$\mathrm{NO}_{\text {[humain] }}+$ boire $+\mathrm{N}_{\text {[quant.] }} \rightarrow$ wypić 
- $\mathrm{N} 0_{[\text {humain] }}+$ boire $+\mathrm{N} 1_{\text {[abstrait/ humain] }}$ (cf. 3.5)

pour la valeur inachevée de boire :

$\mathrm{N} 0_{[\text {humain] }}+$ boire $+\mathrm{N1}_{\text {[abstrait/humain] }} \rightarrow$ pić za

pour la valeur achevée de boire :

$\mathrm{N} 0_{[\text {humain] }}+$ boire $+\mathrm{N} 1_{\text {[abstrait/ humain] }} \rightarrow$ wypić / napić się za

- $\mathrm{N} 0_{\text {[humain] }}+$ boire $+\mathrm{N1}_{\text {[abstrait] }}$ (cf. 3.6)

pour la valeur inachevée de boire :

$\mathrm{N} 0_{[\text {humain] }}+$ boire $+\mathrm{N} 1_{\text {[abstrait] }} \rightarrow$ przepijać

pour la valeur achevée de boire :

$\mathrm{N} 0_{[\text {humain] }}+$ boire $+\mathrm{N} 1_{\text {[abstrait] }} \rightarrow$ przepic

Pour résumer, à la forme imperfective, à côté de pić s'utilisent wypijać (notamment pour une valeur itérative et terminative), et przepijać. À l'aspect perfectif, le choix s'effectue tout d'abord entre wypić et napić się, sélectionnés en fonction du caractère de $\mathrm{N} 1$, de son déterminant, d'une éventuelle présence d'un adjectif épithète à côté de $\mathrm{N} 1$, et du contexte. Nous avons aussi remarqué que le quantitatif qui précède $\mathrm{N} 1$ ou qui se substitue à celui-ci par métonymie bloque napić się. La troisième forme perfective, à l'usage clair et très limité, est przepić. Certes, il existe encore d'autres possibilités, contextuelles, de traduire boire vers le polonais, que nous n'avons pas pris en considération, tenant compte des subtilités des deux langues : française et polonaise, et surtout de la richesse des valeurs aspectuelles de la première et du système développé de préfixation / suffixation de la deuxième. Néanmoins, nous pensons avoir considérablement contribué par notre analyse à la pratique lexicographique bilingue. 


\section{BIBLIOGRAPHIE}

Banyś, W. (2002a). «Bases de données lexicales électroniques - une approche orientée objets. Partie I : Questions de modularité ». Neophilologica, 15, 7-28.

Banyś, W. (2002b). «Bases de données lexicales électroniques - une approche orientée objets. Partie II : Questions de description ». Neophilologica, 15, 206-248.

Cholewa, J. (2008). « Analyse en schèmes sémantico-cognitifs du verbe polonais 'opaść / opadać' ». Neophilologica, 20, 24-45.

Cholewa, J. (2017). Structure conceptuelle et éléments de construction du sens de 'tomber' et de '(-paść / (-)padać'. Białystok: Wydawnictwo Uniwersytetu w Białymstoku.

Declés, J.-P. (2003). "Une classification aspectuelle des schèmes sémantico-cognitifs ». Studia Kognitywne, 5, 53-69.

Declés, J.-P. (2005). « La polysémie verbale, un exemple, le verbe avancer ». In : O. Soutet (ed.), La polysémie. Paris : Presses de l'Université de Paris-Sorbonne, 111-136.

Emirkanian, L. (2008). «Sémantique du verbe monter. Proposition d'un noyau de sens ». In: J. Durand, B. Habert, B. Laks (ed.), Congrès Mondial de Linguistique Française 2008. Paris : Institut de Linguistique Française, 2009-2020. DOI : 10.1051/cmlf08016 (la date du dernier accès : 6.09.2018).

François, J. (2006). «L'espace sémantique comparé des deux verbes français tenir et allemand halten : noyau et extensions ». In: D. Lebaud, C. Paulin, K. Ploog (ed.), Constructions verbales et production de sens. Actes du Colloque, Besançon, 26-28 janvier 2006. Besançon : Presses Universitaires de Franche-Comté, 293-306.

Giermak-Zielińska, T. (1979). Polskie czasowniki przedrostkowe o znaczeniu przestrzennym i ich odpowiedniki w języku francuskim. Wrocław : Zakład Narodowy im. Ossolińskich.

Gross, G. (1999). "Élaboration d'un dictionnaire électronique ». Bulletin de la Société Linguistique de Paris, t. 94, fasc. 1, 113-138.

Gross, G. (2012). Manuel d'analyse linguistique. Lille : Presses Universitaires de Septentrion.

Gross, G. (2015). «Traitement automatique de la polysémie ». Studia Romanica Posnaniensia, $42 / 1,15-33$.

Gross, M. (1975). Méthodes en syntaxe. Paris : Hermann.

Grzegorczykowa, R., Laskowski, R., Wróbel, H. (1998). Gramatyka współczesnego języka polskiego. Morfologia. Warszawa : Wydawnictwo Naukowe PWN.

Hrabia, M. (2014). «Wstęp do obiektowej analizy czasownika aller w konstrukcjach z określnikami temporalnymi dla celów thumaczenia automatycznego francusko-polskiego ». LingVaria, 2 (18), 43-54.

Jalenques, P. (2010). « Analyse sémantique et contraintes distributionnelles : l'exemple du verbe monter ». In : M. Iliescu, H. Siller-Runggaldier, P. Danler (ed.), Actes du XXVe Congrès International de Linguistique et de Philologie Romanes, vol. 3. Berlin / New York : De Gruyter, 115-124.

Janowska, A. (1999). Funkcje przestrzenne przedrostków czasownikowych w polszczyźnie. Katowice : Wydawnictwo Uniwersytetu Śląskiego.

Leeman, D. (2004). " Prémices d'une description du sens du verbe tourner (dans ses emplois intransitifs) ». Linx, 50, 33-52, http://linx.revues.org/136. DOI : 10.4000/linx.136 (la date du dernier accès : 17.09.2018).

Skarżyński, M. (2004). Słownik gniazd słowotwórczych współczesnego języka ogólnopolskiego, t. 3. Kraków : Towarzystwo Wydawnicze « Historia Iagellonica ».

Vandeloise, C. (2007). « Le verbe ALLER. L'affranchissement du contexte d'énonciation immédiat ». Journal of French Language Studies, 17 (3), 343-359, http://journals.cambridge.org/ action/displayJournal?jid=JFL (la date du dernier accès : 15.09.2018). 
Venant, F. (2008). « Représentation géométrique et calcul dynamique du sens lexical : application à la polysémie de livre ». Langages, 172, 30-52.

Victorri, B. (2002). "Espaces sémantiques et représentation du sens ». EcartS, <halshs00009486> (la date du dernier accès : 3.09.2018).

Victorri, B. (2010). "Le localisme à l'épreuve du verbe aller ». Corela, Numéros spéciaux, « Espace, Préposition, Cognition - Hommage à Claude Vandeloise », http://edel.univ-poitiers. fr/corela/document.php?id=2367 (la date du dernier accès : 06.09.2018).

Włodarczyk, A., Włodarczyk, H. (2001). «La préfixation verbale en polonais ». Studia Kognitywne, 4, 93-109.

Wróbel, H. (2001). Gramatyka języka polskiego. Kraków : Spółka Wydawnicza « OD NOWA ».

Yune, K.-Ch. (2011). La sémantique des mots de verticalité en français et leurs emplois métaphoriques : une approche monosémique et cognitive. Thèse de doctorat, Université du Québec à Montréal, http://www.archipel.uqam.ca/4168/ (la date du dernier accès : 4.09.2018).

Żłobińska-Nowak, A. (2008). Désambiguïsation des expressions lexicales des opérateurs de l'espace dans le cadre d'une approche orientée objets : les verbes de mouvement FR monter et sortir et leurs équivalents polonais. Katowice : Wydawnictwo Uniwersytetu Śląskiego.

\section{DICTIONNAIRES ET CORPUS}

Dobrzyński, J., Dutka, A., Frosztęga, B., Kaczuba, I., Karna, J. (1983). Wielki słownik francuskopolski, vol. 2. Warszawa : Wiedza Powszechna.

Dobrzyński, J., Kaczuba, I., Frosztęga, B. (1983). Wielki słownik francusko-polski, vol. 1. Warszawa : Wiedza Powszechna.

Dubois, J., Dubois-Charlier, F. (2013). Les verbes français (Version LVF + 1), http://rali.iro. umontreal.ca/rali/?q=fr/lvf.

Frantext, https://www.frantext.fr/.

Narodowy Korpus Języka Polskiego, http://nkjp.pl/.

Trésor de la Langue Française informatisé, http://atilf.atilf.fr/.

Wielki słownik języka polskiego, http://www.wsjp.pl/. 